\title{
Negative-index metamaterials: is double negativity a real issue for dissipative media?
}

\author{
Thomas Brunet, Olivier Poncelet, and Christophe Aristégui* \\ Univ. Bordeaux, CNRS, Bordeaux INP, UMR 5295 I2M-APY, 33405 Talence, France
}

Received 5 August 2015 / Accepted 19 October 2015

\begin{abstract}
One way to obtain materials with a negative index is to achieve media with doubly negative constitutive parameters. We show that the double negativity required for perfect (non-dissipative) media is not necessary for real passive (lossy) metamaterials, for which single negativity of one of the two constitutive parameters may be sufficient. The analysis is presented through original diagrammatic representations involving both the real and imaginary parts of the dynamic constitutive parameters for isotropic media. A valid demonstration for acoustic and electromagnetic metamaterials is supported by considering an actual soft 3D locally-resonant ultrasonic metafluid having a negative acoustic index while being a single-negative medium. The crucial role of the imaginary parts of the constitutive parameters is then discussed through its impact on the figure of merit. Finally, the analysis is extended to an example of an electromagnetic negative-index metamaterial exhibiting both single and double negativity.
\end{abstract}

Key words: Negative index, Dissipative media, Effective constitutive parameters.

\section{Introduction}

The achievement of negative-index materials (NIMs) for both electromagnetic and acoustic waves has been the subject of intense research for 15 years [1]. A particular recent challenge in the field of metamaterials has been the manufacture of three-dimensional NIMs for optics [2] and acoustics [3]. Although many approaches have been proposed to obtain 3D NIMs, a large part of the metamaterial community has focused its efforts on the fabrication of double-negative materials with simultaneous negative values for the effective constitutive parameters, i.e., relative permittivity and relative permeability $(\varepsilon, \mu)$ in electromagnetism [4] or bulk modulus and mass density $(\kappa, \rho)$ in acoustics [5]. It is common practice in this "double negative approach" to focus only on the real parts of the constitutive parameters that, when simultaneously negative, unconditionally lead to a negative refractive index $(n=-\sqrt{\varepsilon \mu}$ or $n=-\sqrt{\rho / \kappa}) \quad$ [1]. However, these constitutive parameters are obviously complex valued in the presence of dissipation that originates from intrinsic losses within the components and from multiple scattering, particularly for strong resonant scattering. When the imaginary parts of the constitutive parameters are large enough compared with their real counterparts, the medium may exhibit a negative index

*e-mail: christophe.aristegui@u-bordeaux.fr while being a single-negative material, as already observed in optics [6] and acoustics [7].

In acoustics, many single-negative materials have been reported (e.g., for sound insulation) that possess a negative bulk modulus $\kappa$ [8-10] or negative mass density $\rho$ [11-13]. Double-negative acoustic metamaterials with simultaneous negative $\kappa$ and $\rho$ [14-16] have also been proposed to achieve a negative acoustic index [7]. However, although double negativity clearly implies a negative index [5], the opposite is not true [3]. It has been shown in dissipative isotropic homogeneous metamaterials that the $2 \mathrm{D}$ space of constitutive parameters should be carefully considered [17]. In terms of the sign of the real-valued parameters $\kappa$ and $\rho$ [18], the conventional twoaxis diagrammatic representation that is often used to identify NIMs [19] is not appropriate for dissipative media. In this diagrammatic representation, the two quadrants of identical signs of the real parts of the constitutive parameters unambiguously define the positive and negative index regimes of the metamaterials. Nevertheless, for the other two quadrants referring to opposite signs (single negativity), the representation is incomplete because the sign of the real part of the index also depends on the imaginary parts of the constitutive parameters. Therefore, for the sake of completeness and full understanding of the positive/negative-index regimes of dissipative isotropic metamaterials, the (former) four quadrants must be enriched by sub-diagrams invoking the signs of the imaginary parts of 
(a)

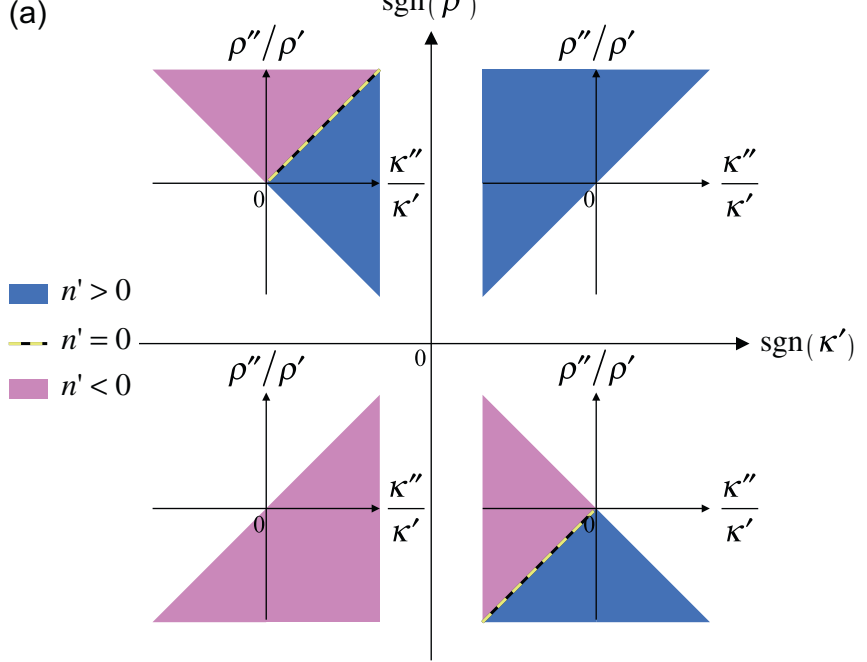

(b)

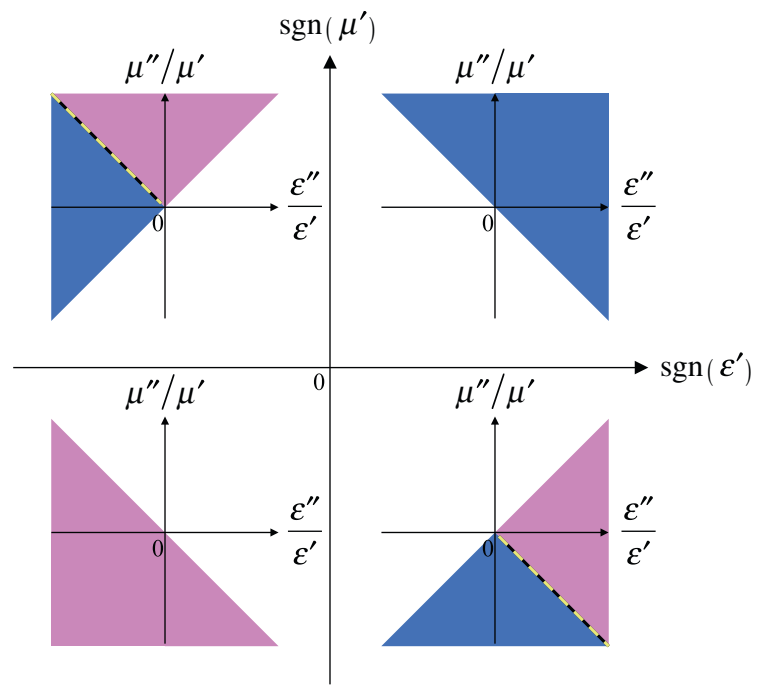

Figure 1. Diagrams identifying the sign of the refractive index according to the signs of the real and imaginary parts of the constitutive parameters. Blue (dark) areas refer to positive-index dissipative media $\left(n^{\prime}>0\right.$ and $\left.n^{\prime \prime}>0\right)$ supporting forward waves, while pink (light) areas refer to negative-index dissipative media $\left(n^{\prime}<0\right.$ and $\left.n^{\prime \prime}>0\right)$ supporting backward waves. White areas refer to amplifying media $\left(n^{\prime \prime}<0\right)$ that are not considered here [17]. (a) Diagram for acoustic metamaterials with complex-valued bulk modulus $\kappa\left(=\kappa^{\prime}+i \kappa^{\prime \prime}\right)$ and mass density $\rho\left(=\rho^{\prime}+\mathrm{i} \rho^{\prime \prime}\right)$. (b) Diagram for electromagnetic metamaterials with complex-valued relative permittivity $\varepsilon\left(=\varepsilon^{\prime}+\mathrm{i} \varepsilon^{\prime \prime}\right)$ and relative permeability $\mu\left(=\mu^{\prime}+\mathrm{i} \mu^{\prime \prime}\right)$.

$\kappa$ and $\rho$. Following on from recent works [17], we propose here insightful diagrammatic representations, based on more readable criteria (valid for acoustics and electromagnetism), for the characterization of dissipative NIMs.

\section{Full views of complex-valued constitutive parameters for dissipative metamaterials}

\subsection{Acoustic metamaterials}

Inhomogeneous media supporting wave propagation are often described through a homogeneous equivalent medium with complex-valued frequency-dependent constitutive parameters. The complex values of these parameters originate from wave-attenuation mechanisms (material dissipation, multiple scattering). For isotropic media, the acoustic refractive index $n$, which is also complex valued $\left(=n^{\prime}+\mathrm{i} n^{\prime \prime}\right)$, is linked to the (effective) complex-valued bulk modulus $\kappa\left(=\kappa^{\prime}+\mathrm{i} \kappa^{\prime \prime}\right)$ and mass density $\rho\left(=\rho^{\prime}+\mathrm{i} \rho^{\prime \prime}\right)$ as follows:

$$
\begin{aligned}
n^{2}= & c_{0}^{2} \frac{\rho}{\kappa}, \\
n= & n^{\prime}+\mathrm{i} n^{\prime \prime}=c_{0} \frac{\sqrt{\rho^{\prime} \kappa^{\prime}}}{|\kappa|} \\
& \times \sqrt{1+\left(\frac{\rho^{\prime \prime}}{\rho^{\prime}}\right)\left(\frac{\kappa^{\prime \prime}}{\kappa^{\prime}}\right)+\mathrm{i}\left\{\left(\frac{\rho^{\prime \prime}}{\rho^{\prime}}\right)-\left(\frac{\kappa^{\prime \prime}}{\kappa^{\prime}}\right)\right\},},
\end{aligned}
$$

where $n^{\prime \prime} \geq 0$ (the passivity condition for harmonic waves with time-dependence $\mathrm{e}^{-\mathrm{i} \omega t}$ ) and $c_{0}$ as the reference phase speed, i.e., the sound speed in the background medium. NIMs therefore refer to media with $n^{\prime}<0$ and are often related with double-negative media [17]. When energy losses can be neglected $\left(\kappa^{\prime \prime} / \kappa^{\prime} \sim \rho^{\prime \prime} / \rho^{\prime} \sim n^{\prime \prime} / n^{\prime} \sim 0\right)$, the acoustic index $n^{\prime}$ is negative for $\kappa^{\prime}<0$ and $\rho^{\prime}<0$ (for example, see Figure 9 in [18]). However, when losses are non-negligible, this simple analysis fails. A clear picture of the constitutive-parameter combinations $(\kappa, \rho)$ ensuring a positive or negative acoustic index in dissipative media has been recently established by analyzing the Poynting vector $\boldsymbol{P}$ (average energy flux density) and the complex-valued wave vector $\boldsymbol{k}\left(=\boldsymbol{k}^{\prime}+\mathrm{i} \boldsymbol{k}^{\prime \prime}\right)$ of the acoustic plane waves propagating in these media [17]. This diagrammatic representation has been reported in Figure 2 of [17] in terms of the phase angles $\theta_{\kappa}$ and $\theta_{\rho}$ of the constitutive parameters $\left(\kappa=|\kappa| \mathrm{e}^{\mathrm{i} \theta_{\kappa}}\right.$, $\left.\rho=|\rho| \mathrm{e}^{\mathrm{i} \theta_{\rho}}\right)$, which are the key values for analyzing the index sign of a given material. To increase the overall readability of the sign of all the different parameters, another type of display involving the ratios $\kappa^{\prime \prime} / \kappa^{\prime}\left(=\tan \theta_{\kappa}\right)$ and $\rho^{\prime \prime} / \rho^{\prime}$ $\left(=\tan \theta_{\rho}\right)$ is proposed. The key criteria for characterizing dissipative NIMs, originally established with $\theta_{\kappa}$ and $\theta_{\rho}$ in [17], can be expressed with the real and imaginary parts of the constitutive parameters $(\kappa, \rho)$ :

$$
\left(\kappa^{\prime}<0 \text { and } \rho^{\prime}<0\right) \text { or }\left(\kappa^{\prime}<0 \text { or } \rho^{\prime}<0 \text {, with } \frac{\rho^{\prime \prime}}{\rho^{\prime}}>\frac{\kappa^{\prime \prime}}{\kappa^{\prime}}\right) .
$$

For positive-index dissipative media, these conditions become:

$$
\left(\kappa^{\prime}>0 \text { and } \rho^{\prime}>0\right) \text { or }\left(\kappa^{\prime}<0 \text { or } \rho^{\prime}<0 \text {, with } \frac{\rho^{\prime \prime}}{\rho^{\prime}}<\frac{\kappa^{\prime \prime}}{\kappa^{\prime}}\right) .
$$

These inequalities provide another viewpoint of the 2D space of the constitutive parameters (Figure 1a). We observe that the acoustic index $n^{\prime}$ may be negative in the singlenegative sub-diagrams $\left(\kappa^{\prime}<0\right.$ or $\left.\rho^{\prime}<0\right)$ as it is in the 


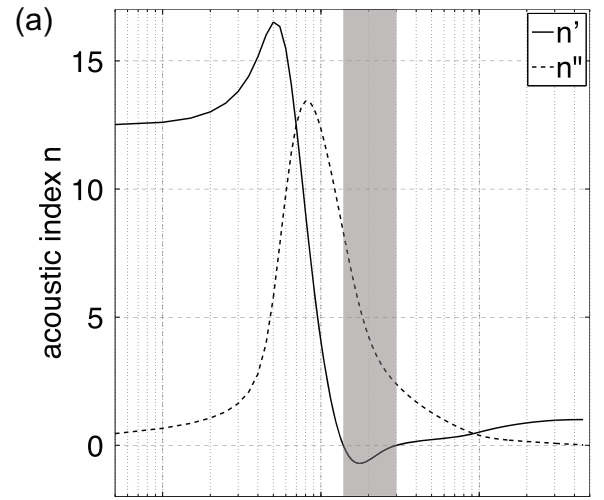

have also been performed [17] for electromagnetic plane waves propagating in dissipative isotropic media. The criteria illustrated in Figure 1 of [17] in terms of the phase angles $\theta_{\varepsilon}$ and $\theta_{\mu}$ of the electromagnetic constitutive parameters can then be expressed in a straightforward manner in terms of the ratios of their imaginary to real parts, $\varepsilon^{\prime \prime} / \varepsilon^{\prime}$ and $\mu^{\prime \prime} / \mu^{\prime}$, as shown in Figure $1 b$. Here also the criteria for characterizing dissipative NIMs from their complex-valued constitutive parameters $(\varepsilon, \mu)$ read:

$$
\left(\varepsilon^{\prime}<0 \text { and } \mu^{\prime}<0\right) \text { or }\left(\varepsilon^{\prime}<0 \text { or } \mu^{\prime}<0 \text {, with } \frac{\mu^{\prime \prime}}{\mu^{\prime}}>-\frac{\varepsilon^{\prime \prime}}{\varepsilon^{\prime}}\right) .
$$
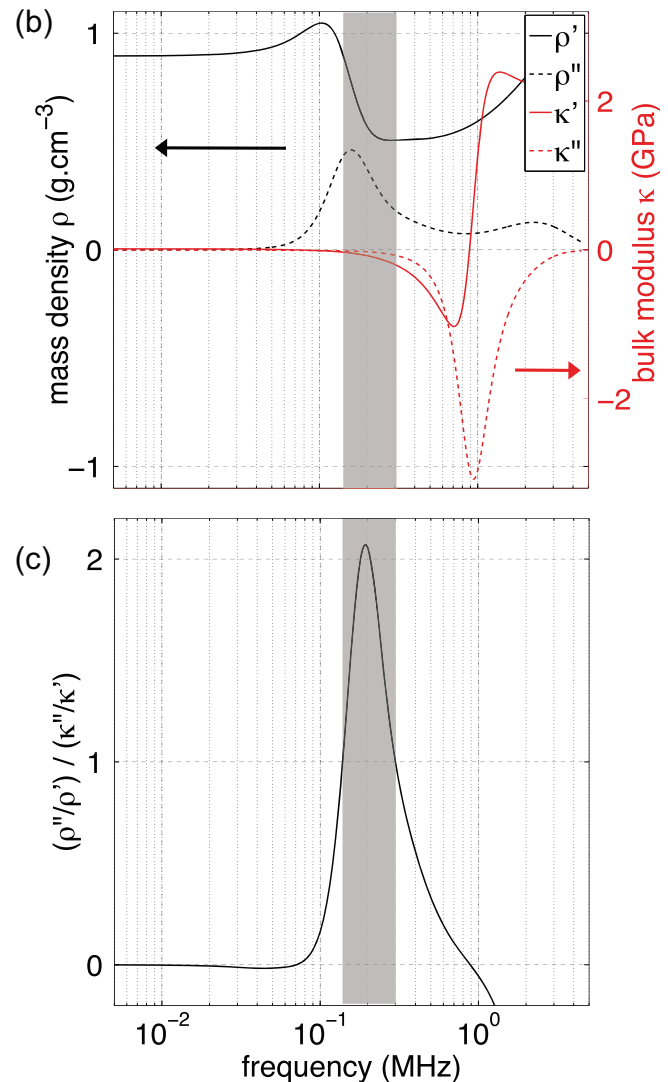

Figure 2. Real and imaginary parts of the dynamic properties for an acoustic metafluid with $20 \%$ of soft porous silicone micro-beads versus the frequency. (a) The acoustic index $n\left(=n^{\prime}+\mathrm{i} n^{\prime \prime}\right)$, (b) the constitutive parameters: bulk modulus $\kappa\left(=\kappa^{\prime}+\mathrm{i} \kappa^{\prime \prime}\right)$ and mass density $\rho\left(=\rho^{\prime}+\mathrm{i} \rho^{\prime \prime}\right)$ and $(\mathrm{c})$ the ratio $\left(\rho^{\prime \prime} / \rho^{\prime}\right) /\left(\kappa^{\prime \prime} / \kappa^{\prime}\right)$. The shaded area $[0.14-0.3 \mathrm{MHz}]$ corresponds to the negative-index frequency band $\left(n^{\prime}<0\right)$.

double-negative sub-diagram $\left(\kappa^{\prime}<0\right.$ and $\left.\rho^{\prime}<0\right)$. Double negativity is clearly not a prerequisite for the emergence of the negative index $\left(n^{\prime}<0\right)$ for dissipative acoustic metamaterials.

\subsection{Electromagnetic metamaterials}

Similar works on the means to deduce the sign of the refractive index $n^{\prime}$ solely from the complex-valued relative permittivity $\varepsilon\left(=\varepsilon^{\prime}+\mathrm{i} \varepsilon^{\prime \prime}\right)$ and relative permeability $\mu\left(=\mu^{\prime}+\mathrm{i} \mu^{\prime \prime}\right)$
For positive-index dissipative media, these conditions become:

$$
\left(\varepsilon^{\prime}>0 \text { and } \mu^{\prime}>0\right) \text { or }\left(\varepsilon^{\prime}<0 \text { or } \mu^{\prime}<0 \text {, with } \frac{\mu^{\prime \prime}}{\mu^{\prime}}<-\frac{\varepsilon^{\prime \prime}}{\varepsilon^{\prime}}\right) .
$$

For $\varepsilon^{\prime \prime}>0$ and $\mu^{\prime \prime}>0$, the conditions (4) and (5) have been found to be consistent with those given in $[20,21]$ and derived only for this combination of $\varepsilon^{\prime \prime}$ and $\mu^{\prime \prime}$. As for acoustic plane waves, the negative-index zone $\left(n^{\prime}<0\right)$ of electromagnetic plane waves cannot be reduced to the double-negative subdiagram $\left(\varepsilon^{\prime}<0\right.$ and $\left.\mu^{\prime}<0\right)$.

\subsection{Diagram correspondences}

Some similarities between Figures 1a and $1 \mathrm{~b}$ can naturally be found by invoking the correspondence between the acoustic and electromagnetic constitutive parameters $\left(\varepsilon \leftrightarrow \kappa^{-1}\right.$, $\mu \leftrightarrow \rho$ ). The equivalence between these two representations addition, by choosing the compressibility $\chi\left(=\kappa^{-1}\right)$ and the mass density $\rho$ as governing parameters for propagating acoustic plane waves, the $2 \mathrm{D}$ space of acoustic parameters can take the same form as that of the electromagnetic parameters displayed in Figure 1b.

\section{Application to real passive metamaterials}

\subsection{Acoustic metamaterials}

The first-ever reported 3D ultrasonic NIM [3] was a metafluid composed of micro-beads made of an "ultra-slow" soft porous silicone rubber [22] randomly dispersed in a host matrix. Its acoustic index $n^{\prime}$ has been shown to be negative over broad frequency ranges, depending on the micro-bead volume fraction $\Phi$. For example, it has been found that $n^{\prime}$ is negative from $0.14 \mathrm{MHz}$ to $0.3 \mathrm{MHz}$ for $\Phi=20 \%$ (see Figure 3d in [3]).

In the present paper, we consider a sample with the same and volume fraction of 20\%). By computing the effective complex-valued acoustic index $n$ for this sample (Figure 2a) [23] as well as the corresponding complex-valued constitutive can then be verified by reversing either axis $\kappa^{\prime \prime} / \kappa^{\prime}$ or $\varepsilon^{\prime \prime} / \varepsilon^{\prime}$. In properties (mean radius of $160 \mu \mathrm{m}$, size dispersion of $25 \%$ 

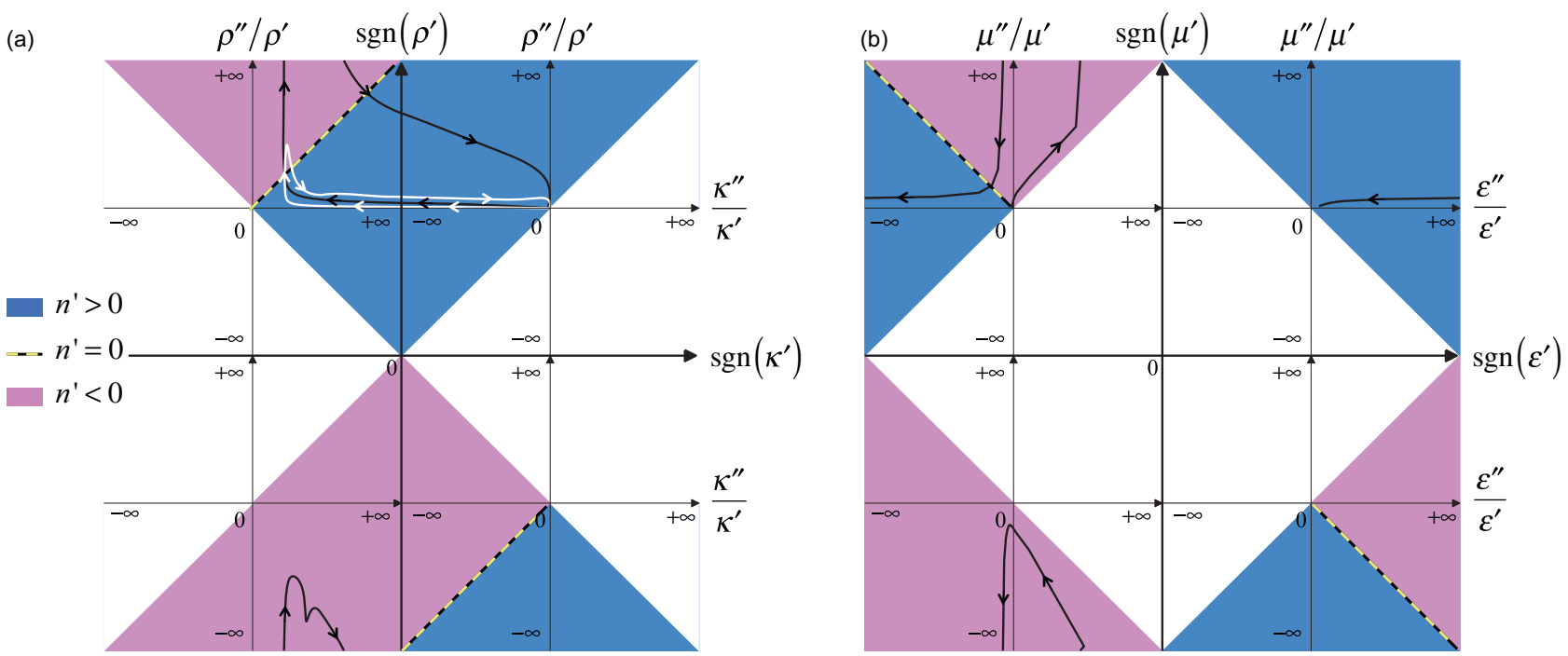

Figure 3. As in Figure 1, the 2D space of the constitutive parameters was explored for (a) acoustic metamaterials [3] with two volume fractions: $\Phi=20 \%$ (white curve) and $\Phi=45 \%$ (black curve) and for (b) electromagnetic metamaterials [30]. Note that a scale function was

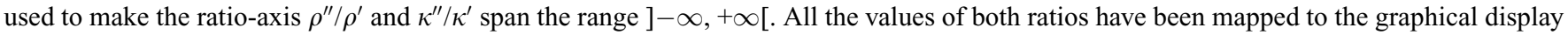
according to the following function: $x^{\prime}=f(x)=x /(1+|x|)$, where $x$ is an actual value of the considered ratio and $x^{\prime}$ is its graphical position proportional to the length of the semi-axis $\left(x^{\prime} \in\right]-1,+1[)$. Thus, the origin $\left(x^{\prime}=0\right)$, the middle $\left(x^{\prime}=1 / 2\right)$ and the edge $\left(x^{\prime}=1\right)$ of the positive semi-axis correspond to ratios $\left(x=\rho^{\prime \prime} / \rho^{\prime}\right.$ or $\left.x=\kappa^{\prime \prime} / \kappa^{\prime}\right)$ equal to 0,1 and $+\infty$, respectively. The same property holds for the negativeratio semi-axis with $x$ and $x^{\prime}$ both negative.

parameters $\kappa$ and $\rho$ (Figure 2b) [24], we find that the negative index is achieved $\left(n^{\prime}<0\right)$ while the material is single negative $\left(\kappa^{\prime}<0\right.$ and $\left.\rho^{\prime}>0\right)$. The change of the $n^{\prime}$ sign, observed in Figure $2 \mathrm{a}$ at 0.14 and $0.3 \mathrm{MHz}$, corresponds to the first bisector $\left(n^{\prime}=0\right)$ of the upper-left sub-diagram in Figure 1a. The values of the ratio $\left(\rho^{\prime \prime} / \rho^{\prime}\right) /\left(\kappa^{\prime \prime} / \kappa^{\prime}\right)$ reported in Figure 2c naturally satisfy the criteria (2) and (3). They are greater than unity when $n^{\prime}<0$ with $\kappa^{\prime}<0$ and $\rho^{\prime}>0$ (single-negative material), as observed from $0.14 \mathrm{MHz}$ to $0.3 \mathrm{MHz}$. Thus, this ratio, coupled with the signs of $\kappa^{\prime}, \kappa^{\prime \prime}, \rho^{\prime}$ and $\rho^{\prime \prime}$, allows the 2D space of complex-valued constitutive parameters to be explored.

The constitutive parameters computed for the metafluid with $20 \%$ of porous micro-beads have been displayed in Figure $3 \mathrm{a}$ (white curve). Each point of the curve corresponds to a pair $(\kappa, \rho)$ computed at a given frequency, and the curve shows the data set with the frequency increasing in the direction of the arrow. The sign of the index $n^{\prime}$ on each of the four sub-diagrams is governed by the signs of $\kappa^{\prime}, \kappa^{\prime \prime}, \rho^{\prime}$ and $\rho^{\prime \prime}$. Transitions from one sub-diagram to another occur each time $\kappa^{\prime}$ or $\rho^{\prime}$ passes through zero, corresponding to infinite values for $\kappa^{\prime \prime} / \kappa^{\prime}$ or $\rho^{\prime \prime} / \rho^{\prime}$. In the quasi-static limit, we begin from the center of the upper-right sub-diagram because the dissipation is zero $\left(\kappa^{\prime \prime}=0\right.$ and $\left.\rho^{\prime \prime}=0\right)$ and the material is double positive $\left(\kappa^{\prime}>0\right.$ and $\left.\rho^{\prime}>0\right)$ for frequencies much lower than the resonance frequencies. From the double positivity observed from 0 to $0.07 \mathrm{MHz}$, the $\kappa^{\prime \prime} / \kappa^{\prime}$ ratio switches from infinitely negative values to infinitely positive values when the $\kappa^{\prime}$ changes sign (crosses the main vertical axis $\left(\kappa^{\prime}=0\right)$ at $0.07 \mathrm{MHz}$ ). For higher frequencies [0.07-0.89 MHz], our metafluid becomes single negative because $\kappa^{\prime}<0$ (while $\rho^{\prime}>0$ remains true), as shown in Figure $2 \mathrm{~b}$. Note that $n^{\prime}$ shifts from positive to negative for an intermediate frequency of $0.14 \mathrm{MHz}$ before reverting to positive for frequencies higher than $0.3 \mathrm{MHz}$, whereas the sample remains single negative $\left(\kappa^{\prime}<0\right)$ until $0.89 \mathrm{MHz}$. Then, at higher frequencies, the sample reverts to being a double-positive material, as it was at low frequencies.

One way to achieve double negativity with these metafluids is to consider a higher micro-bead volume fraction $\Phi$. The constitutive parameters of the metafluid with $\Phi=45 \%$ have been computed according to models developed in $[23,24]$ and displayed in Figure 3a (black curve). As expected, this material is a single- or double-negative NIM according to the range of operating frequencies. Double negativity therefore appears to be unnecessary for obtaining a negative index with dissipative media. This is unlike non-dissipative media, for which single negativity (or positivity) is directly associated with purely evanescent waves, whereas double negativity (or positivity) is associated with non-attenuated propagating waves [18, 19]. One may therefore ask whether double negativity is a real issue for dissipative NIMs.

In the metamaterial community, the challenges for designing NIMs concern more technical issues such as the broadening of negative bands $\left(n^{\prime}<0\right)$ and the decrease of losses $\left(n^{\prime \prime}\right)$ rather than the property of double-negativity. To address the first issue, an increase of the resonator volume-fraction in the metafluid taken as an example seems relevant, as shown in Figure 4a. However, the broadening of the negative bands is accompanied by an increase in wave attenuation. When considering the absolute damping $n^{\prime \prime}$, it is also important to examine the dissipation per wavelength, which is given by the figure of merit $\left(\mathrm{FOM}=\left|n^{\prime} / n^{\prime \prime}\right|\right)$. As depicted in Figure $4 \mathrm{~b}$, the FOM of our acoustic metafluid increases according to the volume fraction while maintaining moderate values $(\sim 1)$. Potential applications, e.g., Pendry's perfect lens [25], require FOMs to be as 


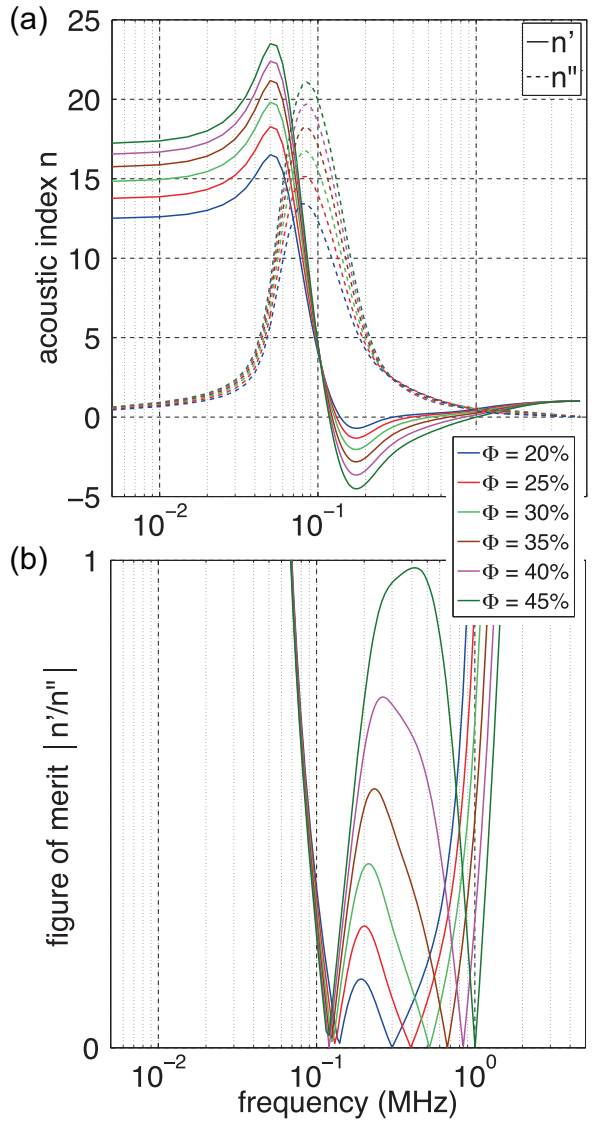

Figure 4. (a) The real and imaginary parts of the acoustic index $n\left(=n^{\prime}+i n^{\prime \prime}\right)$ of an acoustic metafluid with different volume fractions $\Phi$ of soft porous silicone micro-beads with a mean radius of $160 \mu \mathrm{m}$ and a size dispersion of $25 \%$. (b) Corresponding figures of merit (FOM) are defined as the ratio $\left|n^{\prime} / n^{\prime \prime}\right|$.

large as possible. A considerable effort has been made in optics to use gain materials to significantly increase the FOM in NIMs from unity [2] to one hundred [26]. Although few active sonic structures have been recently reported $[27,28]$, this issue is still open to $3 \mathrm{D}$ acoustic material research.

\subsection{Electromagnetic metamaterials}

The issue of the non-equivalence between the negative index and the double negativity of the constitutive parameters has also been addressed in optics. Spectroscopic measurements have revealed negative indices for both single-negative and double-negative metamaterials [29]. Similar to acoustics in the previous section, the $2 \mathrm{D}$ space of complex-valued constitutive parameters is now explored for electromagnetic metamaterials.

As an example, we consider an electromagnetic metamaterial composed of split-ring resonators and wires, as studied by Smith et al. [30]. The frequency-dependent permittivity and permeability of [30] at microwave frequencies are shown in Figures $3 \mathrm{e}$ and $3 \mathrm{f}$ and displayed in Figure $3 \mathrm{~b}$ (black curve). In contrast to Figure 3a, these electromagnetic data begin in the upper-left quadrant (single negativity) at a non-zero frequency, with values of the constitutive parameters corresponding to a quasi-imaginary index $(\mu \sim 1$ and a very large negative value of $\left.\varepsilon^{\prime}\right)$. The index remains mostly imaginary to a frequency of approximately $8 \mathrm{GHz}$ (but still with a small negative real part) and then follows a trajectory in the double-negative area that reverts to the single negative index. Finally, the index becomes positive for the highest available frequencies.

\section{Implications}

Many applications based on either optic or acoustic metamaterials require specific values for the effective refractive index (high, zero or even negative). It is common to target particular extreme values of the dynamic constitutive parameters to obtain these unusual properties. Although only real parts are typically considered, their imaginary counterparts must also be taken into account because they can explain why NIMs do not necessarily require double negativity in their constitutive parameters. These considerations are of the utmost importance for the rational design of passive functional materials.

\section{Conclusions}

Double negativity in electromagnetic or acoustic constitutive parameters is not essential for obtaining metamaterials with negative indices. Although this observation has already been reported for electromagnetic $[6,29]$ and acoustic $[3,7]$ metamaterials, the original diagrammatic approach proposed here allows any metamaterial in the 2D space of complexvalued constitutive parameters for both electromagnetism and acoustics to be located clearly and unambiguously. It aims to quantitatively complete the classical two-axis scheme based on the real parts of the constitutive parameters. We also established a simple form of criteria for the identification of real (dissipative) NIMs according to a ratio involving both the real and imaginary parts of the constitutive parameters. It generalizes the well-known double negativity criterion appropriate for perfect (non-dissipative) NIMs. This analysis may be used for either bulk optical [31] or ultrasonic [32] metamaterials to target specific values of the refractive index without considering single or double negativity.

Acknowledgements. We would like to thank Dr. Stéphane Larouche for supplying the sets of retrieved permitivities and permeabilities published in [30]. This work was supported by the Agence Nationale pour la Recherche (Grant 2011-BS0902101 MetakoustikAerospace Valley) and was performed under the auspices of the Labex AMADEUS ANR-10-LABX-0042-AMADEUS with the help of the French state Initiative d'Excellence IdEx ANR-10IDEX-003-02.

\section{References}

1. C.M. Krowne, Y. Zhang, Physics of negative refraction and negative index materials, Springer, Berlin, 2007.

2. J. Valentine, S. Zhang, T. Zentgraf, et al., Nature 455 (2008) 376. 
3. T. Brunet, A. Merlin, B. Mascaro, et al., Nat. Mater. 14 (2015) 384.

4. C.L. Holloway, E.F. Kuester, J. Baker-Jarvis, P. Kabos, IEEE Trans. Antennas Propag. 51 (2003) 2596.

5. J. Li, C.T. Chan, Phys. Rev. E 70 (2004) 055602.

6. V.M. Shalaev, W. Cai, U.K. Chettiar, et al., Opt. Lett. 30 (2005) 3356.

7. L. Fok, X. Zhang, Phys. Rev. B 83 (2011) 214304.

8. N. Fang, D. Xi, J. Xu, et al., Nat. Mater. 5 (2006) 452.

9. C. Ding, L. Hao, X. Zhao, J. Appl. Phys. 108 (2010) 074911.

10. V.M. Garcia-Chocano, R. Graciá-Salgado, D. Torrent, et al., Phys. Rev. B 85 (2012) 184102.

11. Z. Liu, X. Zhang, Y. Mao, et al., Science 289 (2000) 1734.

12. Z. Yang, J. Mei, M. Yang, et al., Phys. Rev. Lett. 101 (2008) 204301.

13. J. Pierre, B. Dollet, V. Leroy, Phys. Rev. Lett. 112 (2014) 148307.

14. S.H. Lee, C.M. Park, Y.M. Seo, et al., Phys. Rev. Lett. 104 (2010) 054301.

15. X.N. Liu, G.K. Hu, G.L. Huang, C.T. Sun, Appl. Phys. Lett. 98 (2011) 251907.

16. M. Yang, G. Ma, Z. Yang, P. Sheng, Phys. Rev. Lett. 110 (2013) 134301 .

17. J. Dubois, O. Poncelet, C. Aristégui, J. Appl. Phys. 115 (2014) 024902 .
18. M. Kadic, T. Bückmann, R. Schittny, M. Wegener, Rep. Prog. Phys. 76 (2013) 126501.

19. T. Brunet, J. Leng, O. Mondain-Monval, Science 342 (2013) 323.

20. M.W. McCall, A. Lakhtakia, W.S. Weiglhofer, Eur. J. Phys. 23 (2002) 353.

21. R.A. Depine, A. Lakhtakia, Microw. Opt. Technol. Lett. 41 (2004) 315.

22. K. Zimny, A. Merlin, A. Ba, et al., Langmuir 31 (2015) 3215.

23. P.C. Waterman, R. Truell, J. Math. Phys. 2 (1961) 512.

24. C. Aristégui, Y.C. Angel, Wave Motion 44 (2007) 153.

25. J.B. Pendry, Phys. Rev. Lett. 85 (2000) 3966.

26. S. Xiao, V.P. Drachev, A.V. Kildishev, et al., Nature 466 (2010) 735.

27. A. Baz, New J. Phys. 11 (2009) 123010.

28. B.I. Popa, S.A. Cummer, Nat. Comms. 5 (2014) 3398.

29. U.K. Chettiar, A.V. Kildishev, H.K. Yuan, et al., Opt. Lett. 32 (2007) 1671.

30. D.R. Smith, D.C. Vier, Th. Koschny, C.M. Soukoulis, Phys. Rev. E 71 (2005) 036617.

31. P. Baron, A. Iazzolino, K. Ehrhardt, et al., Opt. Mater. Express 11 (2013) 1792.

32. R.M. Guillermic, et al., Abstracts of the 3rd International Conference on Phononic Crystals/Metamaterials, Phonon Transport and Phonon Coupling, Phononics, 2015, p. 280, in press.

Cite this article as: Brunet $\mathrm{T}$, Poncelet $\mathrm{O} \&$ Aristégui $\mathrm{C}$ : Negative-index metamaterials: is double negativity a real issue for dissipative media? EPJ Appl. Metamat. 2015, 2, 3. 